\title{
Aplicación web para la gestión de planes e informes de trabajo académico de profesores para Instituciones de Educación Superior
}

\section{Web application for the management of plans and reports of academic work of teachers for Institutions of Higher Education}

\author{
MACÍAS-BRAMBILA, Hassem Rubén ${ }^{1} \dagger^{*}$, ZAMORA-RAMOS, Victor Manuel $^{2}$, BECERRA- \\ VELÁZQUEZ, Violeta Del Rocío ${ }^{2}$, OSORIO-ANGEL, Sonia ${ }^{2}$ \\ ${ }^{1}$ Universidad de Guadalajara - Sistema de Universidad Virtual, Av. Enrique Díaz de León No. 782, Col. Moderna, \\ Guadalajara, Jalisco, México, C.P. 44190 \\ ${ }^{2}$ Universidad de Guadalajara - Centro Universitario de Ciencias Exactas e Ingenierías, Blvd. Gral. Marcelino García \\ Barragán No. 1421, Colonia Olímpica, Guadalajara, Jalisco, México, C.P. 44430
}

ID 1 Autor: Hassem Rubén, Macías Brambila / ORC ID: 0000-0002-6540-7464, CVU CONACYT ID: 902812

ID $1^{\text {er }}$ Coautor: Victor Manuel, Zamora-Ramos / ORC ID: 0000-0001-9537-6630, CVU CONACYT ID: 903643

ID $2^{\text {do }}$ Coautor: Violeta del Rocío, Becerra-Velázquez / ORC ID: 0000-0003-2866-977X, CVU CONACYT ID: 316236

ID $3^{\text {er }}$ Coautor: Sonia, Osorio-Angel / ORC ID: 0000-0003-4540-4191, CVU CONACYT ID: 967323

DOI: $10.35429 /$ JTAE.2019.7.3.18.25

Recibido: 09 de Enero, 2019; Aceptado 30 de Marzo, 2019

\section{Resumen}

El presente artículo describe el proceso de análisis, diseño, desarrollo e implementación de una aplicación web para la gestión de los planes e informes de trabajo de Profesores de Tiempo Completo (PTC) de Instituciones de Educación Superior (IES), a través de la colaboración del Cuerpo Académico Consolidado (CAC) UTJAL-CA-2 Responsabilidad Social, Sustentabilidad y Desarrollo Integral para PyMES de la Universidad Tecnológica de Jalisco y el Cuerpo Académico en Formación (CAEF) UDG-CA-991 Desarrollo de Competencias Profesionales en Ciencias Computacionales del Centro Universitario de Ciencias Exactas e Ingenierías de la Universidad de Guadalajara. Este proceso se desarrolló a través de la metodología ágil SCRUM por la periodicidad de las fases y su implementación. El objetivo de la aplicación es brindar una solución informática para la gestión de los planes e informes académicos que de acuerdo a las recomendaciones de los Organismos Acreditadores son necesarios como parte de los procesos que aseguran la calidad en los Programas Educativos, contribuyendo en la sistematización y automatización de los procesos de almacenamiento, consulta y validación, facilitando la gestión de los mismos.

Aplicación web, Desarrollo web, Planes de trabajo

\begin{abstract}
This article describes the process of analysis, design, development and implementation of a web application for the management of the plans and work reports of Full Time Teachers (FTT) of Higher Education Institutions (HEI), through collaboration of the Research Group (RG) UTJALCA-2 Social Responsibility, Sustainability and Integral Development for SMEs of the Universidad Tecnológica de Jalisco and the Research Group UDG-CA-991 Development of Professional Competencies in Computer Sciences of the Centro Universitario de Ciencias Exactas e Ingenierías of the Universidad de Guadalajara. This process was developed through the agile SCRUM methodology for the periodicity of the phases and their implementation. The objective of the application is to provide a computer solution for the management of the academic plans and reports that according to the recommendations of the Accreditation Experts are necessary as part of the processes that ensure the quality in the Educational Programs, thus allowing to systematize and automate its processes of storage, consultation and validation, facilitating the management of them.
\end{abstract}

Web application, Web development, Work plans

Citación: MACÍAS-BRAMBILA, Hassem Rubén, ZAMORA-RAMOS, Victor Manuel, BECERRA-VELÁZQUEZ, Violeta Del Rocío, OSORIO-ANGEL, Sonia. Aplicación web para la gestión de planes e informes de trabajo académico de profesores para Instituciones de Educación Superior Revista de Tecnología y Educación. 2019. 3-7: 18-25

\footnotetext{
* Correspondencia del Autor (Correo electrónico: hassem.macias@academico.udg.mx)

$\dagger$ Investigador contribuyendo como primer autor.
} 


\section{Introducción}

La acreditación de los Programas Educativos (PE) es un ejercicio que contempla y visualiza la evaluación como un proceso de mejora continua y como un seguimiento voluntario y sistemático del cumplimiento de las funciones universitarias de una Institución de Educación Superior (IES).

En México, el Consejo para la Acreditación de la Educación Superior (COPAES) define la acreditación como un reconocimiento que realiza una entidad externa a un PE que cumple y trabaja continuamente en el mejoramiento de la calidad de sus procesos, en el cumplimiento de su misión y que satisface un conjunto de indicadores, criterios $y$ estándares enfocados a la calidad y pertinencia, los cuales son establecidos por algún Organismo Acreditador (OA) reconocido por COPAES.

Este proceso de evaluación que realizan los OA esta regulado por un Marco General de Referencia (MGR), el cual de acuerdo a COPAES (2019) determina los lineamientos técnicos-metodológicos para realizar los procesos de acreditación de los PE. Dentro de este MGR se establece la guía de documentos y evidencias que son necesarias para el proceso de acreditación, así como los atributos que deben ser tomados en cuenta para la evaluación, sus indicadores y la definición de cada una de las etapas del proceso.

El proceso de acreditación por un OA inicia con la autoevaluación, en donde el PE, después de manifestar su interés en participar en el proceso, realiza un conjunto de tareas de verificación de los criterios e indicadores establecidos por el OA y el cumplimiento que la IES y el PE dan al Marco de Referencia, a través de la recolección, análisis y presentación de las evidencias pertinentes que corresponden a los atributos, criterios e indicadores establecidos.
En este sentido, y considerando que las IES de México contemplan este proceso de acreditación como parte fundamental de sus Planes de Desarrollo Institucionales (PDI), el Cuerpo Académico Consolidado (CAC) UTJAL-CA-2 de la Universidad Tecnológica de Jalisco, desde sus Líneas de Investigación Aplicada y Desarrollo Tecnológico (LIIADT), junto con el Cuerpo Académico en Formación (CAEF) UDG-CA-991 Desarrollo de Competencias Profesionales en Ciencias Computacionales del Centro Universitario de Ciencias Exactas e Ingenierías de la Universidad de Guadalajara, iniciaron con el desarrollo de una herramienta tecnológica que permitiera coadyuvar en el proceso de acreditación de los PE.

Para el desarrollo de este proyecto, se tomaron en cuenta los Marcos de Referencia del Consejo de Acreditación de la Enseñanza de la Ingeniería, A.C. (CACEI), del Consejo Nacional de Acreditación en Informática y Computación, A.C. (CONAIC) y del Consejo de Acreditación en Ciencias Administrativas, Contables y Afines, A.C. (CACECA).

Dentro de estos Marcos de Referencia, divididos en criterios, el primero de estos de denomina Personal Académico, y en este criterio el PE debe demostrar de acuerdo a CACEI (2018) que los profesionales que participan como académicos son suficientes y pertinentes, que además cuentan con una combinación adecuada de formación académica y profesional, y que tienen una distribución adecuada de actividades sustantivas, además de que son evaluados y apoyados para su superación y que los académicos se involucran en la adecuación del Plan de Estudios del PE.

La necesidad de contar con evidencias, pero sobre todo, con los elementos que permitan generar impactos positivos en la comunidad del PE, se pretende que a través de una aplicación web que permita la gestión de los planes e informes anuales de los profesores del PE, abone directamente a los indicadores de distribución de actividades sustantivas, y que además permita establecer un marco que favorezca la realización de evaluaciones integrales del desarrollo del personal académico, así como un plan de desarrollo o de trabajo dentro del PE, alineando los esfuerzos de vinculación, gestión, docencia, extensión, tutorías e investigación. 


\section{Metodología}

El proyecto se desarrolló en dos periodos semestrales comprendidos en el año 2018 e inició con la selección de la metodología de desarrollo de software, siendo Scrum la determinada, debido a su alta adaptabilidad al cambio y a que permite abordar proyectos completos. Esta metodología esta basada en la realización de entregas parciales de forma regular, y además permite que el desarrollo del proyecto fluya con mayor rapidez.

Scrum establece tres roles principales que definen la conformación de las personas que trabajarán en el proyecto: el Project owner (propietario del proyecto) el cual define o establece los objetivos, además de realizar las verificaciones de las actividades del proyecto, el Master Scrum (experto en Scrum) que resuelve los problemas que surgen durante el desarrollo con el team (equipo), y el Scrum team (equipo Scrum) que esta conformado por el personal enfocado en el desarrollo del proyecto.

Esta metodología es definida por Laínez (2015) como adecuada e ideal para el desarrollo de proyectos incrementales en escenarios complejos, en donde los requisitos suelen cambiar frecuentemente. Esta implementación se trabajó en fases, tales como: análisis, diseño, desarrollo y pruebas.

\section{Análisis}

Esta fase inició con la elaboración del "Product Backlog" (pila de producto). Este documento contiene la recolección de toda la información que es relevante para el proyecto, las tareas que se deberán realizar, los requerimientos que se deben cumplir, así como todas las funcionalidades que el proyecto requiere.

La recolección de requerimientos se generó a través de una serie de entrevistas con los directores de carrera y miembros de los comités de acreditación de los PE de Técnico Superior Universitario en Mecatrónica área Automatización, Tecnologías de la Información y Comunicación área Sistemas Informáticos, Administración área Recursos Humanos, Desarrollo de Negocios área Mercadotecnia y de la Ingeniería en Mecatrónica y la Ingeniería en Desarrollo e Innovación Empresarial de la Universidad Tecnológica de Jalisco.
Así como con los directores de los PE de Ingeniería en Mecatrónica, Ingeniería Civil y la Licenciatura en Terapia Física de la Universidad Politécnica de la Zona Metropolitana de Guadalajara, así como miembros del comité de acreditación de la Ingeniería en Computación del Centro Universitario en Ciencias Exactas e Ingenierías de la Universidad de Guadalajara.

Asimismo; para esta fase se consideró lo establecido en el Marco de Referencia de CACEI, CACECA y CONAIC, además de la estructura establecida en el Curriculum del Programa para el Desarrollo Profesional Docente, para el Tipo Superior (PRODEP), lo que permitió establecer el alcance, enfoque, objetivos, impacto y aplicación para la gestión de los planes e informes anuales.

A partir de lo anterior se determinó el diseño de un sistema a la medida que sea capaz de gestionar de una forma rápida, eficiente y clara el proceso de elaboración, resguardo, evaluación, seguimiento y cierre de los planes de trabajo e informes de los PTC adscritos a los PE.

Después de clasificar y categorizar por funcionalidad la información, se inició con la elaboración del documento de Especificación de Requerimientos de Software (ERS) para lo que se utilizó la plantilla que ofrece el IEEE Std 830-1998 del Institute of Electrical and Electronics Engineers (IEEE, 1998), en este documento se especificaron los roles de los participantes, las características de los usuarios, el alcance del software, así como los requerimientos funcionales y no funcionales. A continuación se muestra la descripción de algunos requerimientos específicos del ERS:

\begin{tabular}{l|l|} 
Número de requisito & R1.11 \\
Nombre de requisito & Crear módulo de configuración de IES \\
\cline { 2 - 2 } Tipo & Requisito $\square$ Restricción \\
\cline { 2 - 2 } Fuente del requisito & Entrevista 28/02/2018, registro 05 \\
Prioridad del requisito & $\bigotimes$ Alta/Esencial $\square$ Media/Deseado $\square$ Baja \\
\hline
\end{tabular}
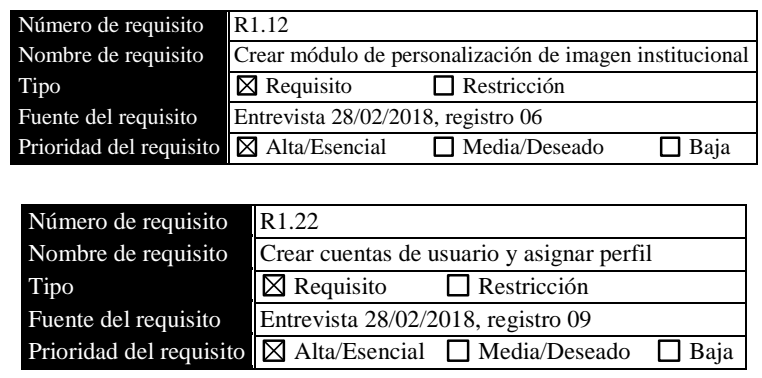

\begin{tabular}{|l|l|}
\hline $\begin{array}{l}\text { Número de requisito } \\
\text { Nombre de requisito }\end{array}$ & Ex4.20 \\
\hline
\end{tabular}

MACÍAS-BRAMBILA, Hassem Rubén, ZAMORA-RAMOS, Victor Manuel, BECERRA-VELÁZQUEZ, Violeta Del Rocío, OSORIOANGEL, Sonia. Aplicación web para la gestión de planes e informes de trabajo académico de profesores para Instituciones de Educación Superior Revista de Tecnología y Educación. 2019 


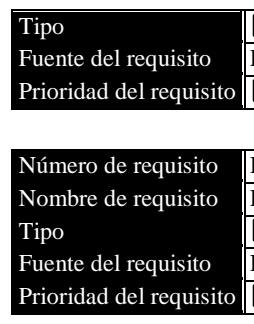

$\triangle$ Requisito $\square$ Restricción Entrevista 02/03/2018, registro 22 $\nabla$ Alta/Esencial $\square$ Media/Deseado $\square$ Baja

R4.22

Exportar informe anual de cumplimiento a PDF $\triangle$ Requisito $\square$ Restricción Entrevista 02/03/2018, registro 25 $\nabla$ Alta/Esencial $\square$ Media/Deseado $\square$ Baja

Tabla 1 Especificación de requerimientos Fuente: Elaboración Propia

La segunda etapa de esta fase es la definición del Sprint Backlog (lista de tareas), documento en el que se definen las tareas que se deben realizar y se asignan a las personas encargadas de ejecutarlas. También se asigna el periodo en el que se debe llevar a cabo cada una de las actividades, para esta tarea de realizó un diagrama de Gantt.

Un Sprint es un periodo determinado de tiempo en el que se realizan acciones que fueron descritas durante el Sprint Backlog. El objetivo de los Sprints es obtener entregas parciales que permitan realizar pruebas en un modelo incremental. Esta etapa contempla la ejecución de reuniones diariamente con los miembros del equipo, donde no deben ser mayor a 15 minutos. Estas reuniones también llamadas Daily Scrum (reunión diaria), incluyen la discusión de todo aquello que se ha realizado, de lo que se planea realizar y de todos aquellos problemas, situaciones, vulnerabilidades o impedimentos que se tuvieron.

\section{Diseño}

Esta fase contempla el diseño arquitectónico de la aplicación y el semántico de datos enfocado a la Base de Datos. El primero incluyó el desarrollo del diagrama de bloques, que ayudo a determinar un bosquejo de los módulos o fragmentos de la funcionalidad y/o modelos de la aplicación, así como la determinación del flujo de datos y las entradas y salidas de los procesos, lo que facilitó el desarrollo del diagrama de clases para ofrecer un mayor nivel de detalle, así como para la determinación de los fragmentos auxiliares necesarios para el funcionamiento; mostrando las clases que componen el sistema, sus atributos, métodos y relaciones pertinentes.

Asimismo; como una buena práctica para el desarrollo y con el objetivo de ofrecer un mayor detalle, se diseñaron las tarjetas Clase-Responsabilidad-Colaboración (CRC), las que describen la naturaleza de cada una de las clases y las relaciones que requieren estar vigentes en el sistema. A continuación se muestran la tarjeta CRC de la clase RetenciónPlan:

\begin{tabular}{|c|c|c|c|}
\hline \multicolumn{4}{|c|}{ Retención Plan } \\
\hline Superclase & Actividad General & Subclase & N/A \\
\hline \multicolumn{2}{|c|}{ Atributos } & \multicolumn{2}{|c|}{ Métodos } \\
\hline \multicolumn{2}{|c|}{$\begin{array}{ll}- & \text { id_actividad_general : INT } \\
- & \text { id_retencion : INT }\end{array}$} & \multicolumn{2}{|c|}{$\begin{array}{ll}- & \text { crear() : bool } \\
- & \text { modificar() : bool } \\
- & \text { mostrar(): } \\
& \text { ActividadGeneral } \\
- & \text { eliminar() : bool }\end{array}$} \\
\hline \multicolumn{2}{|c|}{ Responsabilidad } & \multicolumn{2}{|c|}{ Colaboración } \\
\hline \multicolumn{2}{|c|}{$\begin{array}{l}\text { Relacionar las actividades de } \\
\text { retención al plan de trabajo. }\end{array}$} & \multicolumn{2}{|c|}{ Actividad General. } \\
\hline
\end{tabular}

Tabla 2 Tarjeta CRC Retención-plan Fuente: Elaboración Propia

Se realizó el diagrama de casos de uso para modelar los escenarios en los que el usuario interactúa con el sistema, y de esta manera especificar como es que acceden a distintas funcionalidades, así como determinar las relaciones que existen entre los casos de uso planteados. A continuación se muestra el diagrama de casos de uso de la administración de carreras:

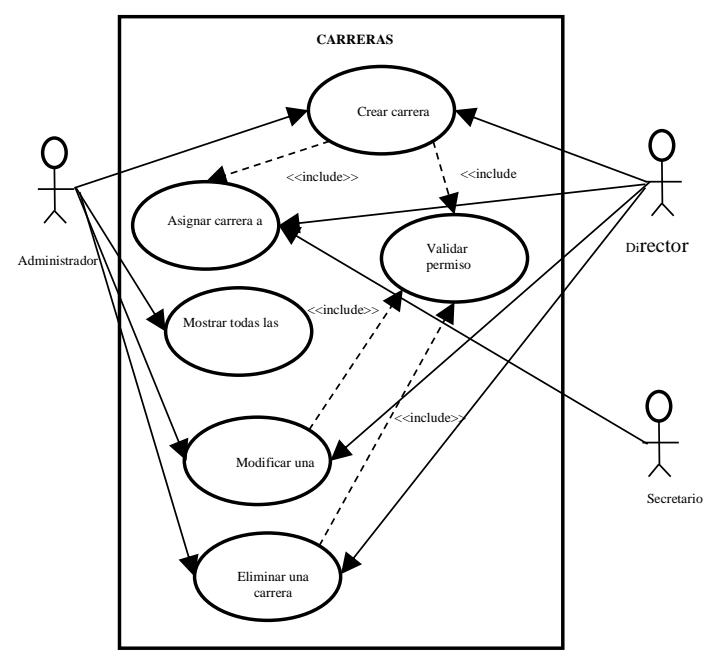

Figura 1 Diagrama de Casos de Uso para la administración de las carreras

Fuente: Elaboración Propia

Estos diagramas establecieron las relaciones y el detalle para la definición de los casos de uso, en los que se describió el proceso, las entidades, precondiciones, postcondiciones, reglas de negocio y las metas. A continuación se muestra el proceso de la definición del caso de uso asignar profesor a una carrera:

\begin{tabular}{|c|c|c|c|}
\hline \# & $\begin{array}{l}\text { Actividad } \\
\text { de actor }\end{array}$ & $\begin{array}{l}\text { Actividad de sistema } \\
\text { [Descripción/Cálculo] }\end{array}$ & [Condición] Alternativa \\
\hline 1 & Presionar & El sistema despliega la & - $\mathrm{Si}$ la conexión \\
\hline
\end{tabular}

MACÍAS-BRAMBILA, Hassem Rubén, ZAMORA-RAMOS, Victor Manuel, BECERRA-VELÁZQUEZ, Violeta Del Rocío, OSORIOANGEL, Sonia. Aplicación web para la gestión de planes e informes de trabajo académico de profesores para Instituciones de Educación Superior Revista de Tecnología y Educación. 2019 


\begin{tabular}{|l|l|l|c|}
\hline & $\begin{array}{l}\text { el botón de } \\
\text { agregar un } \\
\text { profesor }\end{array}$ & $\begin{array}{l}\text { vista de la creación de } \\
\text { usuarios, con todos los } \\
\text { datos a llenar }\end{array}$ & $\begin{array}{l}\text { internet falla, la vista } \\
\text { no puede cargarse }\end{array}$ \\
\hline 2 & $\begin{array}{l}\text { Ingresar } \\
\text { datos }\end{array}$ & $\begin{array}{l}\text { El sistema validará la } \\
\text { información y la } \\
\text { almacenará en la base } \\
\text { de datos }\end{array}$ & $\begin{array}{l}\text { Si no existen carreras } \\
\text { registradas aparecerá } \\
\text { en blanco el combo } \\
\text { box } \\
\text { Si la conexión con la } \\
\text { base de datos falla, el } \\
\text { sistema no almacenará } \\
\end{array}$ \\
& & $\begin{array}{l}\text { la información } \\
\text { Si un campo queda } \\
\text { vacío no se podrá } \\
\text { guardar }\end{array}$ \\
& & & \\
& & $\begin{array}{l}\text { El sistema guardará un } \\
\text { registro en otra tabla } \\
\text { con el código del } \\
\text { director y de carrera }\end{array}$ & \\
\hline 3 &
\end{tabular}

Tabla 3 Extracto de la definición del caso de uso asignar profesor a carrera

Fuente: Elaboración Propia

Asimismo; este diseño incluyó la identificación de los actores, entidades y la comunicación de los objetos, así como la definición de las máquinas de estado para la representación de cada caso de uso, para esto se implementaron modelos del Lenguaje Unificado de Modelado (UML).

El diseño semántico de datos incluyó la elaboración del diagrama entidad relación para la definición lógica de los datos, así como el modelo relacional y la definición de los metadatos que conformarán la estructura de la base de datos. A continuación se muestra un extracto del modelo relacional:

\begin{tabular}{|c|c|}
\hline Grupo & Alumno \\
\hline $\begin{array}{l}\underline{\mathrm{Id}} \\
\text { Id_plan_informe } \\
\text { nombre }\end{array}$ & $\begin{array}{l}\frac{\mathrm{Id}}{I d} \text { grupo } \\
\text { nombre }\end{array}$ \\
\hline Tutoría plan & Gestión plan \\
\hline $\begin{array}{l}\underline{I d} \\
\text { Id_actividad_general } \\
\text { Id_actividad_tutoria }\end{array}$ & $\begin{array}{l}\frac{\mathrm{Id}}{I d \_ \text {actividad }} \\
\text { nombre }\end{array}$ \\
\hline Actividad tutoría & Tipo actividad tutoría \\
\hline $\begin{array}{l}\underline{\mathrm{Id}} \\
\text { Id_tipo_act_tutoría } \\
\text { nombre }\end{array}$ & $\begin{array}{l}\frac{\mathrm{Id}}{\text { Id_plan_informe }} \\
\text { nombre }\end{array}$ \\
\hline
\end{tabular}

Figura 2 Extracto del modelo relacional Fuente: Elaboración Propia
La fase de desarrollo de la aplicación se llevó a cabo con la colaboración y dirección de proyectos modulares en los que participaron estudiantes del PE de Ingeniería en Computación del Centro Universitario de Ciencias Exactas e Ingenierías de la Universidad de Guadalajara, en donde se utilizó Sublime Text, el cual es definido por Ferrer (2015) como un editor de texto y de código fuente.

Este proceso incluyó la implementación del diseño a través del patrón Modelo-VistaControlador (MVC), que de acuerdo a Teniente (2003), contempla la correcta administración de las interacciones que se desarrollan con el usuario, así como la presentación de datos para su correcta interpretación y que el flujo de comunicación con la capa de dominio se realice de forma correcta, en esta se utilizó Zend 2.4 con la versión 7.0 del Hypertext Preprocesor (PHP), definido por Cobo (2005) como un lenguaje de programación del lado del servidor, fue necesario combinar con la versión compatible de Jquery, que de acuerdo a Flanagan (2011) permite simplificar tareas comunes y ocultar las diferencias entre las versiones y navegadores.

De la misma manera, en el marco de desarrollo Zend Framework, que Padilla (2009) define como una colección de paquetes profesionales para el desarrollo web, en un modelo orientado a objetos con un amplio espectro de beneficios.

La alineación de los requerimientos que establece el MVC obligó a descargar la estructura de la aplicación desde el portal web del proveedor, configurando con las características propias del servidor local, lo que facilitó que los desarrolladores utilizarán los manuales, componentes y librerías del modelo de desarrollo web. A continuación se muestra la interfaz del tablero de control de la aplicación, la cual es accesible a través de http://qplannerapp.com/login :

\section{Desarrollo}



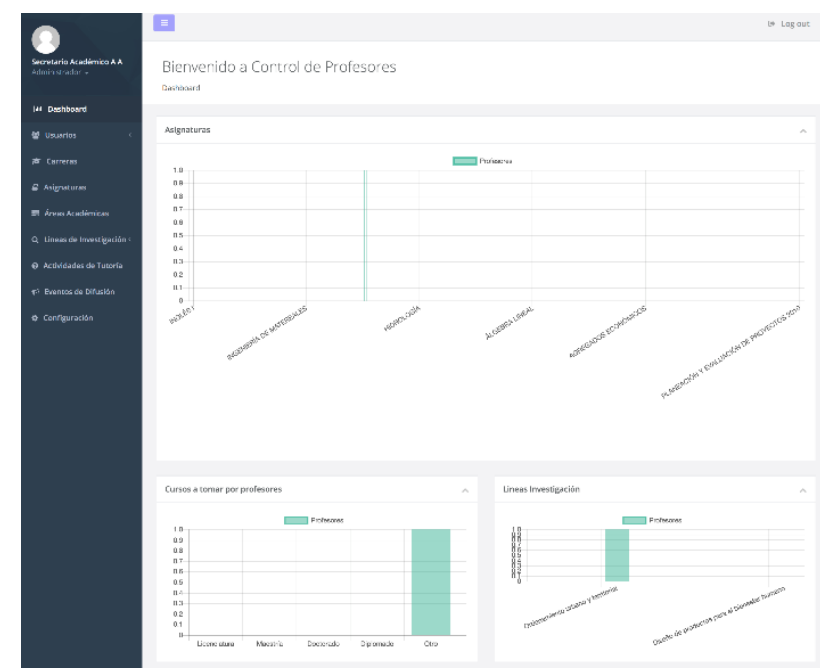

Figura 3 Interfaz del tablero de control Fuente: Elaboración Propia

Desde esta interfaz es posible configurar y operar la aplicación, a través de los módulos de usuarios, carreras, asignaturas, áreas académicas, líneas de investigación, actividades de tutoría, eventos de difusión y configuración. A continuación se muestra la interfaz del módulo de usuarios:

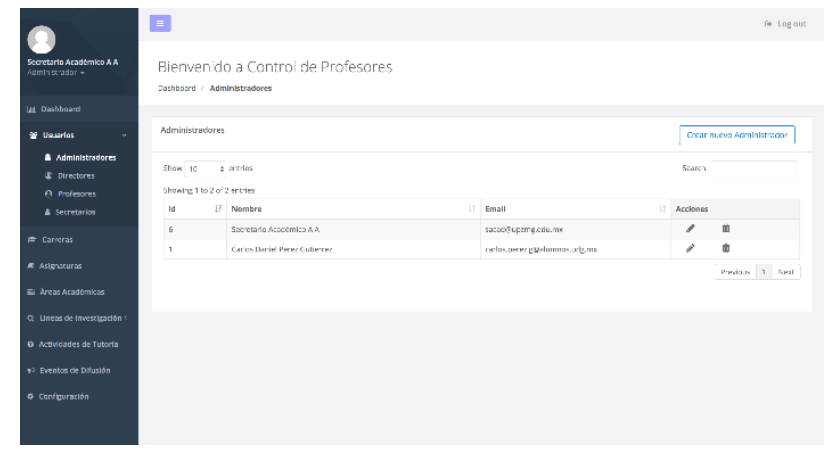

Figura 4 Interfaz del módulo de usuarios

Fuente: Elaboración Propia

Desde este módulo es posible realizar las operaciones de gestión de cuentas de usuarios y perfiles. A continuación se muestra la interfaz de tutorías:

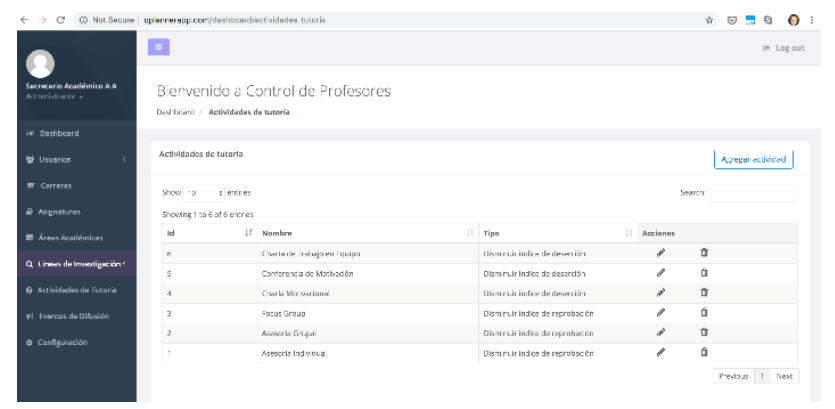

Figura 5 Interfaz del módulo de tutorías Fuente: Elaboración Propia
Desde esta interfaz el profesor puede capturar las acciones de tutoría grupal o individual que están ligadas en su plan de trabajo o en el informe final. A continuación se muestra la interfaz de eventos de difusión:

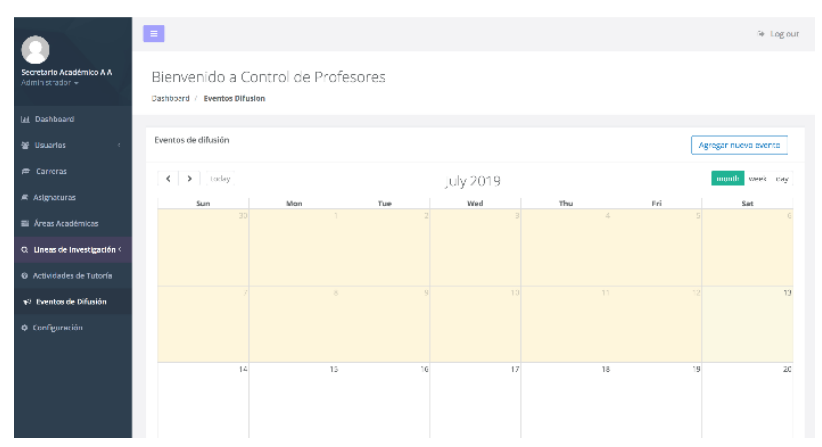

Figura 6 Interfaz del módulo de eventos de difusión Fuente: Elaboración Propia

En este módulo es posible la programación y seguimiento de la participación de los profesores en diversos eventos de difusión a través de su calendarización.

\section{Pruebas}

En esta fase se diseñó e implementó un plan de pruebas para operación de la aplicación de gestión de planes e informes de los PTC.

Este plan de pruebas contempla información general del proyecto, así como un historial de versiones y la definición del alcance de las pruebas, el cual incluyó:

\section{- $\quad$ Elementos de pruebas \\ - Funcionalidades a probar \\ - Pruebas de regresión \\ - Funcionalidades a no probar \\ - $\quad$ Estrategia de pruebas}

Además; con el objetivo de determinar un desempeño aproximado de la ejecución de los Casos de Uso, se definieron a través de los requerimientos funcionales y no funcionales, los criterios de aceptación y rechazo de las pruebas a través de un comportamiento binario. Así como la determinación de los entregables, los recursos, la planeación y la organización para la ejecución del plan de pruebas. 


\section{Resultados}

Se determinó establecer un sistema de almacenamiento de datos centralizado para su administración, debido a que la estimación realizada de acuerdo a la capacidad operativa de una jornada de apertura o cierre de periodos en las seis divisiones de la Universidad Tecnológica de Jalisco de forma concurrente y con un laboratorio de cómputo conectado a la vez por división, contempla una conectividad simultánea de 90 personas aproximadamente, por lo que las peticiones de los clientes, la gestión del almacenamiento de datos y del procesamiento de operaciones, no justifican el costo de una base de datos distribuida. De la misma manera en la Universidad Politécnica de la Zona Metropolitana de Guadalajara se estimó una conectividad simultánea de 20 personas aproximadamente.

En cuanto a la determinación del entorno de desarrollo, de instalación y configuración del servidor no se contempló ningún tipo de restricción salvo la adquisición de licencias, por lo que se optó por la implementación de tecnologías libres, lo que favorece la adaptación de las tecnologías a sus procesos al no representar costos de licenciamiento y que a su vez fue congruente con el resto de módulos que integran el sistema ya existente.

Se realizaron a través del plan de pruebas, casos que contemplaron aspectos de conectividad, rendimiento, interfaz y funcionalidad; para los cuales se comprobó efectivamente la conexión simultánea de 225 usuarios y sus respectivas peticiones como clientes, así como las salidas de los procesos, a través de estadística descriptiva se determina la coincidencia en un $99.02 \%$ con los resultados esperados en los casos de prueba. El resto de las salidas que no cumplieron los criterios de aceptación se documentaron y se reprocesaron.

\section{Conclusiones}

La sistematización del proceso de gestión de los planes e informes de los Profesores de Tiempo Completo en las Instituciones de Educación Superior establece un marco operativo para la administración de los registros que sustentan la distribución de las actividades sustantivas del personal académico.
Esta sistematización es pertinente con el proceso que se requiere cuando un Programa Educativo se somete a cualquier proceso de evaluación, no solo a una acreditación por un Organismo reconocido por COPAES, sino también para los procesos de certificación que algunas IES solicitan al estar certificados en algunas normas ISO.

Este proyecto solo es la primera fase de la sistematización de los criterios de los Marcos Generales de Referencia de COPAES, ya que con los resultados obtenidos, se pretende sistematizar el resto de indicadores y evidencias solicitadas, de tal forma que sea posible proveer una solución informática que solucione las necesidades de todas las IES que se someten a estos procesos.

\section{Agradecimientos}

Agradecemos al Dr. Héctor Pulido González, Rector de la Universidad Tecnológica de Jalisco, por brindar los medios, mecanismos y facilidades para la realización de este proyecto, así como a los Directores de División, Directores de Carrera, Miembros de los Comités de Acreditación, Profesores, Personal de Apoyo y Estudiantes de la Universidad Politécnica de la Zona Metropolitana de Guadalajara y del Centro Universitario de Ciencias Exactas e Ingenierías de la Universidad de Guadalajara que colaboraron en el proyecto.

\section{Referencias}

Aguiar, B. O., Velázquez, R. M., \& Aguiar, J. L. (2019). Innovación docente y empleo de las TIC en la Educación Superior. Revista ESPACIOS, 40(02).

Canós, J. H., Letelier, P., \& Penadés, M. C. (2003). Metodologías ágiles en el desarrollo de software. 1(10), 1-8.

Castrillón, Helder; González Serrano, Carolina \& López, M. (2012). Modelo Arquitectónico para Interoperabilidad entre Instituciones prestadoras de Salud en Colombia. 1-3.

Consejo de Acreditación de la Enseñanza de la Ingeniería, A.C. (2019) Marco de Referencia 2018 del CACEI en el Contexto Internacional (Ingenierías). CACEI. 
Consejo Nacional de Acreditación en Informática y Computación, A.C. (2017) Marco de Referencia para la Acreditación de Programas Académicos de Informática y Computación. CONAIC.

Consejo para la Acreditación de la Educación Superior, A.C. (2016) Marco General de Referencia para los Procesos de Acreditación de Programas Académicos de Tipo Superior. COPAES.

Cortez Roberto (2002) Introducción Al Análisis de Sistemas Y la Ingeniería de Software. Editorial Universidad Estatal a Distancia.

Date, C.J. (2001). Introducción a los sistemas de bases de datos. Pearson Education. 251253.

Davidson, E.J. (1999). Joint application design (JAD) in practice. Journal of Systems and Software. ELSEVIER. 2215-223.

Durango, Alicia. (2014). Diseño web con CSS. Createspace Independent Pub. 11-15.

IEEE. (1998). IEEE Std 830-1998 - IEEE Recommended Practice for Software Requirements Specifications. mayo 20, 2017, de C - IEEE Computer Society Sitio web: https://standards.ieee.org/findstds/standard/8301998.html.

Fowler, Martin \& Kendall Scott. (1999). UML gota a gota. Addison Wesley. 1-5.

Larman, Craug (2003). UML y Patrones. Una introducción al análisis y diseño orientado a objetos y al proceso unificado. Prentice Hall.

Macías Brambila, Hassem R., López Laguna, Ana B., González del Castillo, Edgardo E., \& Tolosa Carrillo, Esaú. (2017). Servidor de aplicaciones como evidencia para sinergia academia-empresa MyPyMES de México. Revista de Tecnología Informática. Ecorfan. 39-43.

Macías Brambila, Hassem R., López Laguna Ana B., Peña Montes de Oca, Adriana I., \& Álvarez Jiménez, Hugo R. (2017). Web Development: Evidence of follow-up for compliance with the UN Global Compact in Construction Companies. Journal-Republic of Paraguay. Ecorfan. 20-26.
Minguet, P. A., \& Solís, A. U. (2019). Educación y Sostenibilidad en la Universidad de Valencia: construyendo futuro desde el pasado. Revista de Educación Ambiental y Sostenibilidad, 1202-1202.

Pilone, D., Pitman, N. (2005). UML 2.0 in a Nutshell. O’Reilly Media, Inc.

Romero J. Especificación de Requisitos Software según el estándar IEEE 830. Recuperado el día 8 de agosto de 2017 de: https://www.fdi.ucm.es/profesor/gmendez/docs/ is0809/ieee830.pdf

Ruiz, I. I. B. (2019). Learning Analytics como cultura digital de las universidades: Diagnóstico de su aplicación en el sistema de educación a distancia de la UNAM basado en una escala compleja. Revista Iberoamericana de Educación, 80(1), 89-116.

Raventós, Pepita (2012). Repositorios digitales: aplicación del modelo OAIS y los esquemas de metadatos a la conservación del patrimonio documental archivístico. Universidad de Lleida. $1-3$.

Sommerville, I. (2011). Software Engineering (9 ed.). Person.

Terrazas Pastor, Rafael (2011). Planificación y programación de operaciones. Scielo. 8-11.

Tuya, Javier; Ramos Roman, I.\& Dolado Cosín, J. (2007). Técnicas cuantitativas para la gestión en la ingeniería de software. Netbiblio. 49-53. 